\title{
Congenital muscular dystrophy with fatty liver and infantile-onset cataract caused by TRAPPC11 mutations: broadening of the phenotype
}

\author{
Wen-Chen Liang ${ }^{1,3 \dagger}$, Wenhua Zhu ${ }^{5,6,7 \dagger}$, Satomi Mitsuhashi, ${ }^{5,6^{*}}$, Satoru Noguchi $i^{5,6}$, Michael Sacher ${ }^{8,9}$, \\ Megumu Ogawa ${ }^{5}$, Hsiang-Hung Shih 1,3, Yuh-Jyh Jong ${ }^{1,2,4,10}$ and Ichizo Nishino 5,6
}

\begin{abstract}
Background: Transport protein particle (TRAPP) is a multiprotein complex involved in endoplasmic reticulum-to-Golgi trafficking. Zebrafish with a mutation in the TRAPPC11 orthologue showed hepatomegaly with steatosis and defects in visual system development. In humans, TRAPPC11 mutations have been reported in only three families showing limb-girdle muscular dystrophy (LGMD) or myopathy with movement disorders and intellectual disability.

Methods: We screened muscular dystrophy genes using next-generation sequencing and performed associated molecular and biochemical analyses in a patient with fatty liver and cataract in addition to infantile-onset muscle weakness.

Results: We identified the first Asian patient with TRAPPC11 mutations. Muscle pathology demonstrated typical dystrophic changes and liver biopsy revealed steatosis. The patient carried compound heterozygous mutations of a previously reported missense and a novel splice-site mutation. The splice-site change produced two aberrantly-spliced transcripts that were both predicted to result in translational frameshift and truncated proteins. Full-length TRAPPC11 protein was undetectable on immunoblotting.

Conclusion: This report widens the phenotype of TRAPPC11-opathy as the patient showed the following: (1) congenital muscular dystrophy phenotype rather than LGMD; (2) steatosis and infantile-onset cataract, both not observed in previously reported patients; but (3) no ataxia or abnormal movement, clearly indicating that TRAPPC11 plays a physiological role in multiple tissues in human.
\end{abstract}

Keywords: Transport protein particle (TRAPP), Endoplasmic reticulum-to-Golgi trafficking, Steatosis, Cataract, Congenital muscular dystrophy

\section{Background}

Transport protein particle (TRAPP) is a multiprotein complex involved in endoplasmic reticulum (ER)-toGolgi trafficking and possibly other membrane trafficking steps $[1,2]$. Oligomerization of TRAPP can give rise to complexes with variable components in any one of

\footnotetext{
* Correspondence: satomi11@me.com

${ }^{\dagger}$ Equal contributors

${ }^{5}$ Department of Neuromuscular Research, National Institute of Neuroscience, National Center of Neurology and Psychiatry, Tokyo, Japan

${ }^{6}$ Department of Genome Medicine Development, Medical Genome Center,

National Center of Neurology and Psychiatry, Tokyo, Japan

Full list of author information is available at the end of the article
}

several positions and might allow for a combinatorial diversification of TRAPP function, perhaps regulating cell-specific activities [3]. A loss-of-function mutation in the zebrafish TRAPPC11 orthologue is characterized by hepatomegaly with steatosis, thereby named foie gras mutant, and by defects in visual system development $[4,5]$. In human, there has been only one report of TRAPPC11 mutations, describing one Syrian family with limb-girdle muscular dystrophy (LGMD) phenotype, which was labeled LGMD2S, and two families of Hutterite ancestry with myopathy phenotype, movement disorders and intellectual disability [6]. In addition to impaired TRAPP assembly and disrupted Golgi apparatus 
architecture, alterations of the lysosomal membrane glycoproteins lysosome-associated membrane protein 1 (LAMP1) and LAMP2 were also observed in the cells of affected individuals, suggesting a defect in the transport of secretory proteins as the underlying pathomechanism. We herein report the first Asian patient carrying compound heterozygous mutations in the TRAPPC11 gene who developed congenital muscular dystrophy (CMD) phenotype with prominent fatty liver and infantile-onset cataract, further broadening the clinical phenotype of TRAPPC11-opathy.

\section{Case presentation}

\section{Clinical and pathological features}

The currently 8-year-old Han Chinese girl residing in Taiwan, born to non-consanguineous parents, was found unable to stand up at age 1 year. Timeline of the developmental milestones before then was not recalled by the parents. She started walking independently at age 1 year and 6 months and readily fell down. At age 2 years, speech delay was noticed as she could not speak any significant single word though she could understand and follow simple orders. Bilateral cataracts were also found at the same age. Her birth history was uneventful, and there was no relevant family history. However, rehabilitation did not show marked improvement in her speech and motor functions. At age 3 years and 6 months, high levels of transaminases were found immediately prior to cataract surgery. Subsequently high creatine kinase (CK) level was identified resulting in a referral to a pediatric myologist. Physical examination showed mild lordosis, positive Gowers' sign with waddling gait, and decreased deep tendon reflexes, as well as hepatomegaly. Neither ataxia nor abnormal movement was observed. Blood biochemistry indicated that the levels of AST $(180 \mathrm{IU} / \mathrm{L}$; normal <40), ALT (1577 IU/L; normal <40), and serum CK $(8699$ IU/L; normal: <175) were markedly elevated. Further assessment identified borderline cognitive function (Bayley-II: mental developmental quotient score
$(D Q)=82-87$; motor $D Q=67)$. Brain magnetic resonance imaging (MRI) did not show cerebral or cerebellar atrophy, and there was no structural abnormality except for slightly reduced periventricular white matter volume with angular-shaped ventricles (Fig. 1a). On muscle $\mathrm{CT}$, posterior compartment of lower extremities were preferentially involved and gluteal muscles were severely affected (Fig. 1b).

Due to persistent high levels of AST/ALT and hepatomegaly, liver biopsy was performed and revealed excessive lipid accumulation in hepatic cells, suggestive of steatohepatitis (Fig. 2a). Muscle biopsy of the biceps brachii revealed dystrophic change with scattered necrotic and regenerating fibers and moderate endomysial fibrosis (Fig. 2b) and mildly increased lipid droplets in the cytoplasm of muscle fibers on oil red $\mathrm{O}$ staining compared to age-matched control (Fig. 2c). No notable abnormality was shown on immunohistochemistry using the antibodies against C-terminal, N-terminal, and core domain of dystrophin (Novocastra Lab.), alpha-, beta-, delta-, and gamma- sarcoglycans(Novocastra Lab.), alpha-(Upstate) and beta-dystroglycans(Novocastra Lab.), merosin(Chemicon International), collagen $\mathrm{VI}(\mathrm{ICN}$ Biomedicals, Inc), dysferlin(Novocastra Lab.), caveolin-3(Transduction Lab.), and emerin(Novocastra Lab.). In the subsequent 4-year follow-up, her muscle weakness remained stationary. To date, no cardiac or respiratory problems were found.

\section{Molecular and protein analyses}

In order to identify the cause of this syndrome, targeted next-generation sequencing covering reported muscular dystrophy related genes (AGRN, ALG13, ANO5, B3GALNT2, B3GNT1, CAPN3, CAV3, CHKB, COL12A1, COL6A1, COL6A2, COL6A3, DAG1, DES, DMD, DNAJB6, DOK7, DOLK, DPAGT1, DPM1, DPM2, DPM3, DYSF, EMD, FAT1, FHL1, FKRP, FKTN, FLNC, GFPT1, GMPPB, ISPD, ITGA7, KLHL9, LAMA2, LARGE, LMNA, MEGF10, MICU1, MYOT, PLEC, POMGNT1, POMGNT2, POMT1, POMT2, PTRF, SGCA, SGCB, SGCD, SGCG, POMK,
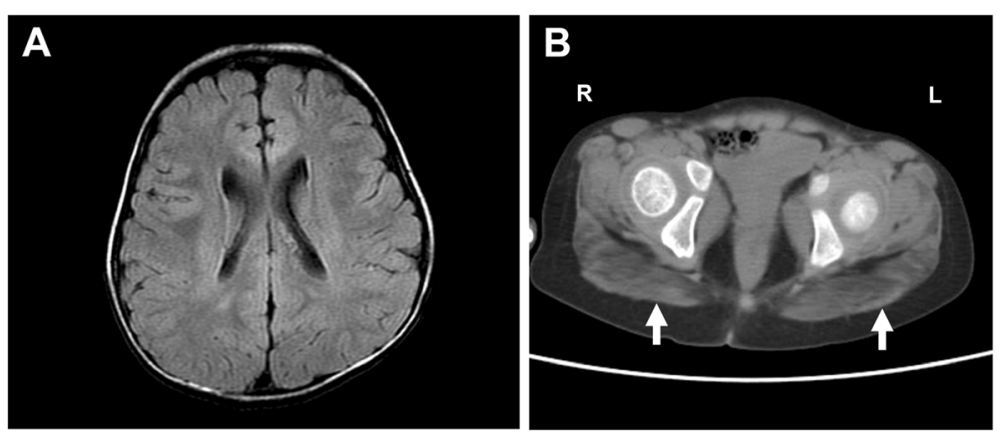

Fig. 1 Brain and muscle imaging. a Mildly reduced periventricular white matter volume with angular-shaped ventricles was shown on brain MRI (T2-weighted flair). b Gluteal muscles were most affected on muscle CT as the arrows indicate. (R right, L left) 

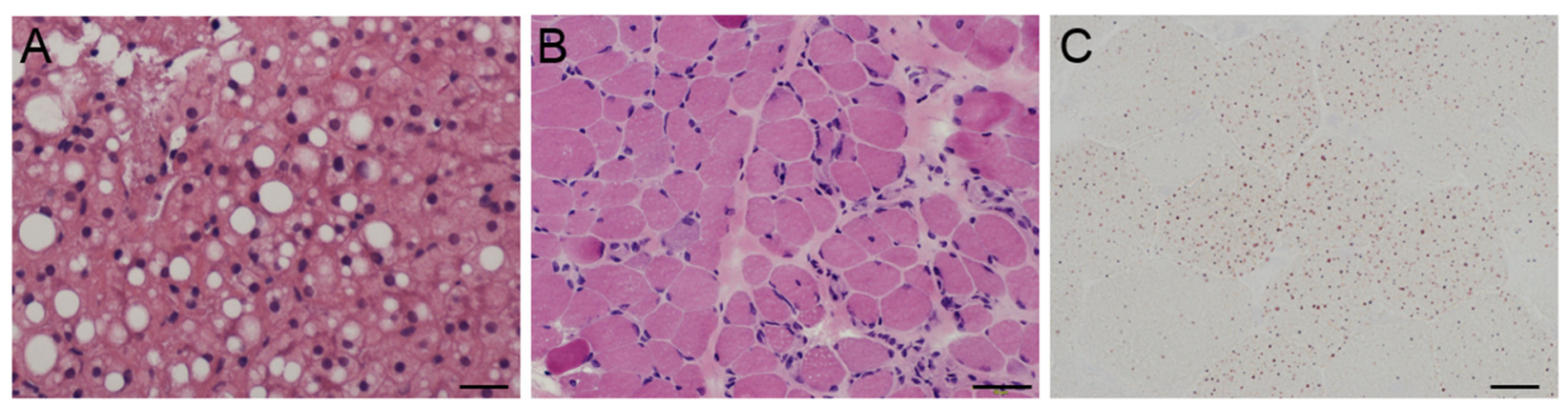

Fig. 2 Liver and muscle pathology. a Hematoxylin and eosin staining (H\&E) of liver biopsy showed marked lipid accumulation consistent with steatohepatitis. (size bar $20 \mu \mathrm{m}$ ) H\&E and oil red O staining of the biopsied muscle revealed dystrophic change (b) and mild lipid accumulation (c). (size bar $50 \mu \mathrm{m}$ for $\mathbf{b}$ and $20 \mu \mathrm{m}$ for $\mathbf{c}$ )

A
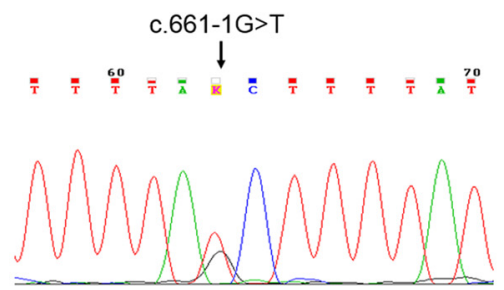

B

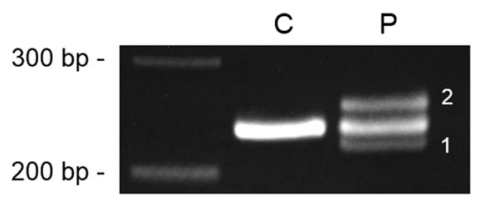

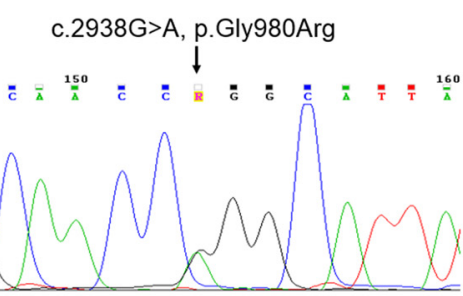

D

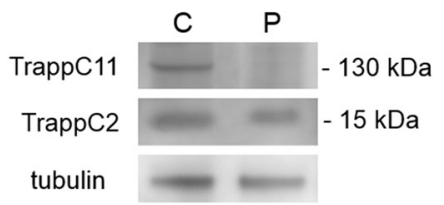

$C_{\text {mutant } 1}$

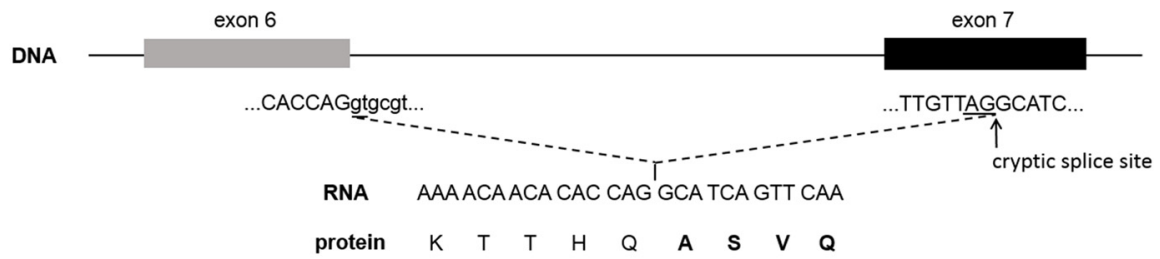

mutant 2

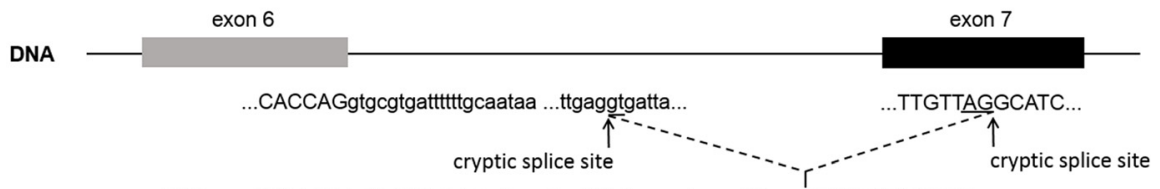

RNA AAAACAACA CAC CAG gtg cgt gat ttt ttg caa taa ...ttttgagGCATCAGTTCAA

protein $K \quad T \quad T \quad H \quad Q \quad V \quad R \quad D \quad F \quad L \quad Q^{*}$

Fig. 3 Molecular and protein analyses. a Sanger sequencing confirmed the compound heterozygous mutations c.661-1G > T and c.2938G > A in TRAPPC11. b Analysis of skeletal muscle cDNA flanking exons 6 and 7 of TRAPPC11 showed two mutant transcripts (1 and 2) in addition to the 234-bp normal amplicon. c The novel c.661-1G > T splice-site mutation results in two mutant transcripts; mutant 1 with a truncated exon 7, and mutant 2 with both truncated exon 7 and a cryptic exon in intron 6, both of which are predicted to cause translational frameshift, p.Leu240Alafs* 10 and p.Leu240Valfs*7, respectively. Altered amino acids are in bold. $\mathbf{d}$ Protein analysis using the biopsied muscle revealed the absence of TRAPPC11 protein at $130 \mathrm{kDa}$ while TRAPPC2 and tubulin were comparable to control muscle 
SMCHD1, STIM1, SYNE1, SYNE2, TCAP, TMEM43, TMEM5, TNPO3, TRAPPC11, TRIM32) was performed on genomic DNA extracted from blood lymphocytes of the proband. Multiplex primer pools were designed using Ion AmpliSeq $^{\mathrm{Tm}}$ Designer software (Life Technologies). This custom gene panel covers $96.8 \%$ of the coding sequence region of these genes. Enrichment of exonic sequences was performed with Ion AmpliSeq ${ }^{\text {tm }}$ Library Kit 2.0 (Thermo Scientific) and sequenced on an IonPGM $^{\text {ma }}$ (Thermo Scientific) according to the manufacturer's protocol. Compound heterozygous mutations of c.2938G > T (p.Gly980Arg) and c.661-1G > T in TRAPPC11 (NM_021942.5) were identified. Subsequent Sanger sequencing of genomic DNA was performed in both the proband and her parents to confirm the detected variants (Fig. 3a). This revealed that c.2938G > T was found in the father and c.661-1G > T in the mother. Since the c.661-1G > T mutation suggested there might be a splicing defect, further cDNA analysis with SuperScript VILO Master Mix Kit (Life Technologies) for the c.661-1G > T mutation was carried out to investigate illegitimate splicing. The forward primer in exon 5 (5' - TTGTTTGTACTGCCGCACAC-3') and the reverse primer flanking the end of exon 7 and the beginning of exon 8 (5'- GGTCCTATAATTCTTCAGCGCATT-3') generate a 234-bp amplicon from the wild-type TRAPPC11 CDNA sequence. RT-PCR products were then cloned into the pCR4 TOPO-TA vector (Invitrogen) to allow detection of all mRNA products by sequencing with fluorescent dideoxy chain terminators (Applied Biosystems, Foster
City, CA, USA) on an ABI 3130 sequencing instrument (Applied Biosystems). Two additional aberrantly spliced transcripts were identified, and both were predicted to result in translational frameshift (Fig. 3b, c). In biopsied muscle, immunoblotting procedures using anti-TRAPPC11 (1:500), anti-TRAPPC2 (1:500), and antitubulin (1:1000, DM1A, Sigma) as primary antibodies were performed (the TRAPPC11 and TRAPPC2 antibodies were noncommercial and raised against a peptide derived from the carboxy-terminal region of TRAPPC11 and full-length His-tagged TRAPPC2) [3, 7]. Full-length TRAPPC11 protein was not observed (Fig. 3d).

\section{Discussion}

We have demonstrated that the novel splice-site mutation c.661-1G > T results in two different aberrant transcripts, predicted to produce two truncated proteins. The absence of a full-length TRAPPC11 protein by Western blot analysis suggests the possibility that Gly980Arg mutation may destabilize the protein, which was also shown in the previous report describing Gly980Arg in a homozygous manner, or may enhance its degradation [6].

In the previous study, the affected individuals with TRAPPC11 mutations presented with two groups of clinical manifestations: one with more prominent muscular and skeletal symptoms and the other with microcephaly, hyperkinetic movements, ataxia, and intellectual disability, apparently reflecting the difference of the two genotypes, Gly980Arg and Ala372_Ser429del. Three

Table 1 Comparison of the present patient and previously reported patients with TRAPPC11 mutations

\begin{tabular}{|c|c|c|c|}
\hline & c.2938G > A homo a & c. $1287+5 G>$ A homo $^{a}$ & c.2938G > A/c.661-1G > T \\
\hline Number of patient & 3 & 5 & 1 \\
\hline Age of onset & Early school age & Early childhood onset & Around 1-year-old or even earlier \\
\hline Muscle symptoms & Proximal weakness, myalgia, cramps & Mild weakness and hypotonia $(2)^{b}$ & Proximal weakness, hypotonia \\
\hline Muscle pathology & Myopathic $(1)^{b}$ & Myopathic $(2)^{b}$ & Dystrophic \\
\hline CK (IU/L) & $600 \sim 2800$ & $300 \sim 1000$ & $6000 \sim 9000$ \\
\hline Head circumference & Within normal limit & $<3$ rd percentile $(4)^{\mathrm{b}}$ & $(-)$ \\
\hline Intellectual disability & $(-)$ & $(+)$ & Borderline \\
\hline Ataxia & $(-)$ & $(+)$ & $(-)$ \\
\hline Choreiform movement & $(-)$ & $(+)$ & $(-)$ \\
\hline \multirow[t]{2}{*}{ Other neurological problems } & \multirow[t]{2}{*}{$(-)$} & Generalized seizure $(1)^{\mathrm{b}}$ & \multirow[t]{2}{*}{$(-)$} \\
\hline & & abnormal EEG $(2)^{b}$ & \\
\hline Neuroimaging & Not available & Mild cerebral atrophy $(2)^{b}$ & Reduced white matter volume \\
\hline Cardiac involvement & Enlarged right ventricle $(1)^{\mathrm{b}}$ & $(-)$ & $(-)$ \\
\hline Skeletal involvement & Hip dysplasia, scoliosis & Limb asymmetry $(1)^{b}$ & Lordosis \\
\hline Ocular involvement & Esotropia and myopia $(1)^{\mathrm{b}}$ cataract $(1)^{\mathrm{b}}$ & Exophoria, anisometropia, and amblyopia (1) ${ }^{\mathrm{b}}$ & Infantile_-onset cataract \\
\hline Hepatic involvement & $(-)$ & $(-)$ & Steatosis \\
\hline
\end{tabular}

apreviously reported mutation (Ref 6)

${ }^{\mathrm{b}}$ The number of patient (if no number is indicated for the item, it means all patients presented with this feature Homo homozygosity, EEG electroencephalogram 
patients in one family carried homozygous Gly980Arg, and five affected members from two unrelated families had homozygous Ala372_Ser429del. No notable interfamiliar difference of phenotype was observed between the two families with Ala372_Ser429del mutation. Noteworthily, only one patient with Gly980Arg mutation was reported to develop mild cataracts after school age. Clinical manifestation of the patient in the present study is different from the previously reported patients in several respects. First, the patient in the present study presented with steatosis and very early-onset cataract, similar to what was seen in the foie gras mutant in zebrafish. Second, the patient developed a CMD phenotype rather than the LGMD seen in the previous study. Finally, the patient does not display choreiform movement, ataxia, nor any skeletal abnormality. These differences may partly be explained by a more deleterious effect of the splice-site mutation on the TRAPPC11 protein which we expect to lead to complete loss of function, compared to Gly980Arg or a 58 amino acid in-frame deletion in homozygosity in the previous study. Table 1 summarizes the phenotypic differences between the present patient and previously reported patients with TRAPPC11 mutations.

It is noteworthy that, although the patient presented here did not have microcephaly, abnormal involuntary movements, nor cerebral atrophy, which were previously reported in the patients with TRAPPC11 mutations [6], her brain MRI at the age of 3 years and 6 months revealed slightly reduced white matter volume. Reduced white matter volume in pediatric patients is usually associated with periventricular leukomalacia, the major substrate of neurologic deficits in premature infants [8]. However, it might also be the consequence of diffuse axonal damage or maldevelopment such as hypomyelination, which may not be easily differentiated by imaging without serial studies $[9,10]$. Regarding the normal maternal pregnancy and birth history of the patient in this study, ischemic/hypoxic injury-causing white matter volume loss seems unlikely. As the T1- and T2-weighted images did not show notably abnormal intensity, mild hypomyelination was thus considered. Interestingly, the deficiency of a Golgi-associated protein, dymeclin, was recently reported to cause postnatal microcephaly, hypomelination, and ER-to-Golgi trafficking defects in both mice and humans [11]. Although dymeclin has not yet known to be a binding partner of TRAPP complex, the similar Golgi-associated nature and clinical phenotype are indicative of probable interaction and common pathomechanism of these two proteins. The present study also provides further supportive evidence of the relationship between the impaired cellular trafficking and brain phenotype in TRAPPC11-associated disease.

\section{Conclusions}

Collectively, this study widens the phenotype of TRAPPC11-opathy. Although the detailed mechanism causing intracellular lipid storage in liver is still unknown, the phenotype of the patient in this study clearly indicates that TRAPPC11 plays a physiological role in multiple tissues in humans including the liver, muscle, eye, brain, and bone. This may be due to impairment of TRAPPC11 functions in multiple membranetrafficking pathways or other processes.

\section{Consent}

Written informed consent was obtained from the patient's mother for publication of this Case Report and any accompanying images. A copy of the written consent is available to Editors of this journal on request.

\section{Abbreviations}

TRAPP: transport protein particle; LGMD: limb-girdle muscular dystrophy; ER: endoplasmic reticulum; LAMP: Iysosome-associated membrane protein; CMD: congenital muscular dystrophy; CK: creatine kinase.

\section{Competing interests}

The authors declare that they have no competing interests.

\section{Authors' contributions}

WCL and WZ have made substantial contributions to conception and design of the study and acquisition, analysis, and interpretation of the data and have been involved in drafting the manuscript and revising it critically for important intellectual content. SM has made substantial contributions to conception and design of the study and acquisition, analysis, and interpretation of the data, has been involved in revising the manuscript critically for important intellectual content, and agrees to be accountable for all aspects of the work in ensuring that questions related to the accuracy or integrity of any part of the work are appropriately investigated and resolved. SN has made substantial contributions to conception and design of the study and analysis and interpretation of the data and has been involved in revising the manuscript critically for important intellectual content. MS has made substantial contributions to analysis and interpretation of the data and have been involved in revising the manuscript critically for important intellectual content. MO has made substantial contributions to acquisition, analysis, and interpretation of the data. HHS has made substantial contributions to acquisition, analysis, and interpretation of the data. YJJ has made substantial contributions to acquisition, analysis, and interpretation of the data and has been involved in revising the manuscript critically for important intellectual content. IN has made substantial contributions to interpretation of the data and has been involved in revising the manuscript critically for important intellectual content. All authors have read and approved the final version of the manuscript.

\section{Acknowledgements}

This study was supported partly by Intramural Research Grant (26-7, 26-8) for Neurological and Psychiatric Disorders of NCNP, Health and Labour Sciences Research Grants for Comprehensive Research on Persons with Disabilities (H25-Shinkei Kin-Ippan-004) and Practical Research Project for Rare/Intractable Diseases (H26-Itaku (Nan)-Ippan-081) from Japan Agency for Medical Research and Development, AMED. WZ is supported by the State Scholarship Fund from China Scholarship Council (CSC_201406105045). MS is supported by the Canadian Institutes of Health Research, the Natural Sciences and Engineering Research Council of Canada, and the Canada Foundation for Innovation, and is a member of the Groupe de Recherche Axé sur la Structure des Protéines (GRASP) network.

\section{Author details}

${ }^{1}$ Departments of Pediatrics, Kaohsiung Medical University Hospital, Kaohsiung Medical University, Kaohsiung, Taiwan. '2Department of Laboratory Medicine, Kaohsiung Medical University Hospital, Kaohsiung Medical University, 
Kaohsiung, Taiwan. ${ }^{3}$ Department of Pediatrics, School of Medicine, College of Medicine, Kaohsiung Medical University, Kaohsiung, Taiwan. ${ }^{4}$ Graduate Institute of Medicine, College of Medicine, Kaohsiung Medical University, Kaohsiung, Taiwan. ${ }^{5}$ Department of Neuromuscular Research, National Institute of Neuroscience, National Center of Neurology and Psychiatry, Tokyo, Japan. ${ }^{6}$ Department of Genome Medicine Development, Medical Genome Center, National Center of Neurology and Psychiatry, Tokyo, Japan. ${ }^{7}$ Department of Neurology, Huashan Hospital, Fudan University, Shanghai, China. ${ }^{8}$ Department of Biology, Concordia University, Montreal, QC H4B 1R6, Canada. ${ }^{9}$ Department of Anatomy and Cell Biology, McGill University, Montreal, QC H3A 2B2, Canada. ${ }^{10}$ Department of Biological Science and Technology, College of Biological Science and Technology, National Chiao Tung University, Hsinchu, Taiwan.

Received: 10 July 2015 Accepted: 7 August 2015

Published online: 28 August 2015

\section{References}

1. Kim YG, Raunser S, Munger C, Wagner J, Song YL, Cygler M, et al. The architecture of the multisubunit TRAPP I complex suggests a model for vesicle tethering. Cell. 2006;127:817-30.

2. Wendler F, Gillingham AK, Sinka R, Rosa-Ferreira C, Gordon DE, Franch-Marro X, et al. A genome-wide RNA interference screen identifies two novel components of the metazoan secretory pathway. EMBO J. 2010;29:304-14.

3. Scrivens PJ, Noueihed B, Shahrzad N, Hul S, Brunet S, Sacher M. C4orf41 and TTC-15 are mammalian TRAPP components with a role at an early stage in ER-to-Golgi trafficking. Mol Biol Cell. 2011;22:2083-93.

4. Sadler KC, Amsterdam A, Soroka C, Boyer J, Hopkins N. A genetic screen in zebrafish identifies the mutants vps 18, nf2 and foie gras as models of liver disease. Development. 2005;132:3561-72.

5. Gross JM, Perkins BD, Amsterdam A, Egaña A, Darland T, Matsui II, et al. Identification of zebrafish insertional mutants with defects in visual system development and function. Genetics. 2005;170:245-61.

6. Bögershausen N, Shahrzad N, Chong JX, von Kleist-Retzow JC, Stanga D, Li Y, et al. Recessive TRAPPC11 mutations cause a disease spectrum of limb girdle muscular dystrophy and myopathy with movement disorders and intellectual disability. Am J Hum Genet. 2013:93:181-90.

7. Scrivens PJ, Shahrzad N, Moores A, Morin A, Brunet S, Sacher M. TRAPPC2L is a novel, highly conserved TRAPP-interacting protein. Traffic. 2009;10:724-36.

8. Haynes RL, Billiards SS, Borenstein NS, Volpe JJ, Kinney HC. Diffuse axonal injury in periventricular leukomalacia as determined by apoptotic marker fractin. Pediat Res. 2008;63:656-61.

9. Alam GCA, Sahu WCS. Magnetic resonance imaging in evaluation of periventricular leukomalacia. MJAFI. 2010;66:374-80.

10. Steenweg NE, Vanderver A, Blaser S, Bizzi A, de Koning TJ, Mancini GMS, et al. Magnetic resonance imaging pattern recognition in hypomyelinating disorders. Brain. 2010;133:2971-82.

11. Dupuis N, Fafouri A, Bayot A, Kumar M, Lecharpentier T, Ball G, et al. Dymeclin deficiency causes postnatal microcephaly, hypomelination and reticulum-to-Golgi trafficking defects in mice and humans. Hum Mol Genet. 2015;24:2771-83.

\section{Submit your next manuscript to BioMed Central and take full advantage of:}

- Convenient online submission

- Thorough peer review

- No space constraints or color figure charges

- Immediate publication on acceptance

- Inclusion in PubMed, CAS, Scopus and Google Scholar

- Research which is freely available for redistribution 